\title{
Loop-tree duality from vertices and edges
}

\author{
William J. Torres Bobadilla \\ Max-Planck-Institut für Physik, Werner-Heisenberg-Institut, \\ 80805 München, Germany \\ E-mail: torres@mpp.mpg.de
}

ABSTRACT: The causal representation of multi-loop scattering amplitudes, obtained from the application of the loop-tree duality formalism, comprehensively elucidates, at integrand level, the behaviour of only physical singularities. This representation is found to manifest compact expressions for multi-loop topologies that have the same number of vertices. Interestingly, integrands considered in former studies, with up-to six vertices and $L$ internal lines, display the same structure of up-to four-loop ones. The former is an insight that there should be a correspondence between vertices and the collection of internal lines, edges, that characterise a multi-loop topology. By virtue of this relation, in this paper, we embrace an approach to properly classify multi-loop topologies according to vertices and edges. Differently from former studies, we consider the most general topologies, by connecting vertices and edges in all possible ways. Likewise, we provide a procedure to generate causal representation of multi-loop topologies by considering the structure of causal propagators. Explicit causal representations of loop topologies with up-to nine vertices are provided.

KeYwords: NLO Computations, QCD Phenomenology

ARXIV EPRINT: 2102.05048 


\section{Contents}

1 Introduction 1

2 Loop-tree duality formalism 3

2.1 Notation 3

2.2 Maximal loop topology 4

3 Causal representation of Feynman integrands $\quad 6$

3.1 Next-to Maximal and Next-to-Next-to Maximal loop topologies 6

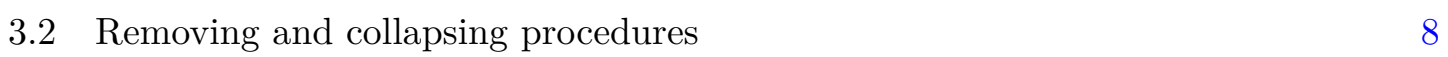

$\begin{array}{lll}3.2 .1 & \text { Removing } & 8\end{array}$

$\begin{array}{llr}3.2 .2 & \text { Collapsing } & 9\end{array}$

4 Five-vertex topologies and beyond $\quad 11$

$\begin{array}{lll}4.1 & \text { Causal representation of five-vertex topologies } & 11\end{array}$

$\begin{array}{lll}4.2 & \text { Causal propagators beyond five vertices } & 14\end{array}$

5 Causal representation of loop topologies without LTD 16

$\begin{array}{llr}6 & \text { Conclusions } & 21\end{array}$

\section{Introduction}

In the recent years, the calculation of physical observables, relevant for high-energy physics colliders, turns everyday to be more challenging due to the high precision the current experiments are demanding $[1,2]$. The Next-to-Leading Order (NLO) revolution, originated by extremely clever ideas, opened a street, based on formal aspects of scattering amplitudes, to provide several phenomenological calculations. In effect, the various ingredients that need to be taken into account were understood and automated in several frameworks [3-9]. Since many of these methods promote the space-time dimension from four to $d$, new reformulations that aim at a local cancellation without altering the dimension are yet under study [10-28].

In view of the Next-to-Next-to-Leading Order (NNLO) revolution, many ingredients have to be ready to properly get combined and also allow for a synergy with the results delivered by the experiments. Among the several obstacles that prevent the NNLO revolution, we would like to emphasise the role in the evaluation of multi-loop Feynman integrals that is drawing the attention in the scientific community [29-33] where the number of kinematic invariants starts increasing. Nonetheless, analytic or numerical evaluation of dimensionally regulated multi-loop Feynman integrals with several internal masses is still a bottleneck that must be sorted out [34-46]. 
In light of providing an alternative approach to the numerical evaluation of multi-loop Feynman integrals, we elaborate on the loop-tree duality (LTD) formalism [47-51]. The LTD formalism, based on the Cauchy residue theorem, can be realised as an operation that opens loops into trees and, due to the way how it is formulated, it is always possible to integrate out the energy component of the loop momenta in any multi-loop Feynman integral. As a byproduct of this operation, one passes from Minkowski to Euclidean space, allowing in this way to compactify all the physical information in spatial components. In other words, a $d$-dimensional Feynman integral in Minkowski space, after making use of the LTD formalism, is expressed only in terms of the spatial components of the loop momenta. In fact, the use of the LTD formalism allows to bypass the standard parametrisation of Feynman integrals in terms of the two Symanzik polynomials (see [52] and references therein) and permits to integrate in Euclidean space. Several phenomenological applications by using LTD, performed at one and two loops [53-57], allow for a reformulation of this formalism [58-62] and motivate alternative studies [63-69].

Recently, it has been observed that the application of the LTD formalism on multiloop Feynman integrands leads to a causal representation displaying only physical information $[58,59]$. In effect, these integrands, written in terms of causal propagators, allow for a smooth numerical evaluation, avoiding in this way numerical instabilities, as discussed in [60]. An alternative approach to our causal representation of Feynman integrands was also presented in ref. [66] by means of $\mathrm{H}$ surfaces, which, within our framework, corresponds to causal propagators.

Inspired by the compact representation found in ref. [60], for the multi-loop Maximal (MLT), Next-to Maximal (NMLT) and Next-to-Next-to Maximal ( $\mathrm{N}^{2} \mathrm{MLT}$ ) loop topology configurations, it is desirable to understand the reason of the same structure of the causal representation, regardless of the loop order. To this end, in this paper, we classify the loop topologies according to the number of vertices and edges. For instance, an $\mathrm{N}^{k} \mathrm{MLT}$ configuration will have $k+2$ vertices, and the number of edges will correspond to the number of connections between vertices in a topology. Besides, we elaborate on the most general topology generated from a given number of vertices and all possible edges. The motivation of doing so is to obtain the simplest and most compact formulae for the causal representation of the loop topology, in such a way that topologies made of less number of vertices and edges can be generated from the latter one by applying certain procedures.

Furthermore, to relate loop topologies through vertices and edges, we introduce two procedures: removing and collapsing. The former procedure removes edges by keeping fixed the number of vertices, whereas the latter collapses a vertex by removing it as well as the edges it is connected. These two procedures allows to generate any kind of topology built from a given number of vertices and allows for a validation of our new results. Then, with the pattern displayed by these procedures in the causal representation, we provide the most general formulae for topologies with up-to nine vertices. The latter, in the most general case, corresponds to a topology at twenty-eight loops.

This paper is organised as follows. In section 2, we recap the main features of the LTD formalism, briefly review the causal representation of the MLT configuration, and introduce the notation in terms of vertices and edges. Then, in section 3, with the new notation, 
we provide the causal representation of NMLT and $\mathrm{N}^{2} \mathrm{MLT}$ and introduce the procedures: removing and collapsing. In section 4, from the knowledge of the former sections, we generate the most general topology of five vertices and apply the removing and collapsing procedures. Besides, we provide a general procedure to generate the causal representation of any loop topology. Then, with the findings of sections 3 and 4 , in section 5 , we provide causal representations of topologies with up-to nine vertices, by only considering relations among causal propagators. Finally, in section 6, we draw our conclusions and discuss the future research directions.

\section{Loop-tree duality formalism}

\subsection{Notation}

In this section, we set the notation and recap the main features of the representation of the loop-tree duality (LTD) formalism by the application of nested residues. Since the main idea of LTD is opening loops into connected trees, let us start the discussion with a generic $N$-point integral at $L$ loops, in the Feynman representation,

$$
\mathcal{A}_{N}^{(L)}(1, \ldots, r)=\int_{\ell_{1}, \ldots, \ell_{L}} d \mathcal{A}_{N}^{(L)}(1, \ldots, r),
$$

where, to simplify the notation in the integration measure, we define $\int_{\ell_{s}} \equiv-\imath \mu^{4-d} \int d^{d} \ell_{s} /(2 \pi)^{d}$ and,

$$
d \mathcal{A}_{N}^{(L)}(1, \ldots, r)=\mathcal{N}\left(\left\{\ell_{i}\right\}_{L},\left\{p_{j}\right\}_{N}\right) \times G_{F}(1, \ldots, r),
$$

in which, the integral is understood as an $L$-loop topology with $r$ internal lines. The numerator $\mathcal{N}$ is a function of the loop and external momenta and is given by the Feynman rules of the theory. The function $G_{F}$ collects all the Feynman propagators of the topology,

$$
G_{F}(1, \ldots, r)=\prod_{i \in 1 \cup 2 \cdots \cup r}\left(G_{F}\left(q_{i}\right)\right)^{\alpha_{i}}
$$

where $\alpha_{i}$ are the powers of the propagators and,

$$
G_{F}\left(q_{i}\right)=\frac{1}{q_{i}^{2}-m_{i}^{2}+\imath 0}=\frac{1}{\left(q_{i, 0}+q_{i, 0}^{(+)}\right)\left(q_{i, 0}-q_{i, 0}^{(+)}\right)},
$$

the usual Feynman propagator of a one single particle, with $m_{i}$ its mass, $+\imath 0$ the infinitesimal imaginary Feynman prescription and,

$$
q_{i, 0}^{(+)}=\sqrt{\boldsymbol{q}_{i}^{2}+m_{i}^{2}-\imath 0}
$$

the on-shell energy of the loop momentum $q_{i}$ written in terms of the spatial components $\boldsymbol{q}_{i}$. Let us remark that the dependence on the energy component of the loop momenta $q_{i, 0}$ is completely pulled out. This is done, in order to apply the Cauchy residue theorem and integrate out one degree of freedom: the energy component of each loop momentum. Hence, when taking the nested residue [62], one selects the poles with negative imaginary part in the complex plane of the energy component of the loop momentum that is integrated, as extensively explained in ref. [59]. 
Hence, the dual representation of the integrand (2.2), after setting on shell the propagators that depend on the loop momentum $q_{i_{1}}$, is defined as follows,

$$
\mathcal{A}_{D}^{(L)}(1, \ldots, r) \equiv \sum_{i_{1} \in 1} \operatorname{Res}\left(d \mathcal{A}_{N}^{(L)}(1, \ldots, r), \operatorname{Im}\left(q_{i_{1}, 0}\right)<0\right)
$$

where the factor $-2 \pi \imath$ that comes from the Cauchy residue theorem is absorbed in the definition of the integration measure as shall be noted in the following. This residue corresponds to integrating out the energy components of the loop momenta. Thus, we can introduce the nested residue as,

$$
\mathcal{A}_{D}^{(L)}(1, \ldots, s ; s+1, \ldots, r) \equiv \sum_{i_{s} \in s} \operatorname{Res}\left(\mathcal{A}_{D}^{(L)}(1, \ldots, s-1 ; s, \ldots, r), \operatorname{Im}\left(q_{i_{r}, 0}\right)<0\right),
$$

where the iteration goes until the $s$-th set and corresponds to setting simultaneously $L$ lines on shell. The latter is equivalent to open the loop topology (or amplitude) into connected trees.

Finally, with the integration of the energy component of the loop momentum, one passes from Minkowski to Euclidean space. In the following, we use the abbreviation,

$$
\int_{\vec{\ell}_{s}} \bullet \equiv-\mu^{d-4} \int \frac{d^{d-1} \ell_{s}}{(2 \pi)^{d-1}} \bullet
$$

for the $(d-1)$-momentum integration measure.

\subsection{Maximal loop topology}

The novel representation of the LTD formalism has displayed very interesting properties at integrand level. In particular, the causal structure of integrands allows for a smooth numerical evaluation and, therefore, a numerical integration. In this section, we briefly recall the main results obtained in $[59,60]$ w.r.t. the Maximal loop topology and set the notation for the following sections.

Interestingly, the LTD formalism has been observed to work at the level of vertices, where the causal thresholds are generated, and is independent of the number of loops. Namely, the structure of the integrand, after cancellations at intermediate steps, manifest the same causal structure independently on the number of internal lines, provided that the number of vertices remains the same.

Let us review the simplest topology generated with two vertices, ${ }^{1}$ the Maximal loop topology (MLT), originally studied in [59], whose causal representation, after applying the LTD theorem, is,

$$
\mathcal{A}_{\mathrm{MLT}}^{(L)}=-\int_{\ell_{1}, \ldots, \ell_{L}} \frac{1}{x_{L+1}}\left(\frac{1}{\lambda_{1}^{+}}+\frac{1}{\lambda_{1}^{-}}\right),
$$

with $x_{s}=\prod_{i=1}^{s} 2 q_{i, 0}^{(+)}$.

\footnotetext{
${ }^{1}$ These kinds of diagrams with $L$ internal lines are usually called multi-loop sunrise or multi-banana diagram.
} 
In eq. (2.9), it is understood an $L$-loop two-point function, with the explicit dependence of the energy of the external momentum $p_{1}$ in the causal propagator $\lambda_{1}^{ \pm}=q_{(1, \ldots, L+1)}^{(+)} \pm p_{1,0}$, with $q_{(1, \ldots, L+1)}^{(+)}=\sum_{i=1}^{L+1} q_{i, 0}^{(+)}$. Here, and in the following, we use $\lambda_{i}^{ \pm}$to refer to the $i$-th causal propagator, with " \pm " the direction flow w.r.t. the external momenta, as discussed below. All causal propagators are expressed as sums of $q_{i, 0}^{(+)}, q_{i_{1}, 0}^{(+)}+q_{i_{2}, 0}^{(+)}+\ldots+q_{i_{r}, 0}^{(+)}$, allowing for a smooth numerical evaluation of the integrand. Let us remark that the application of LTD on a Feynman integrand generates independent terms, obtained from eq. (2.7), that are contaminated by non-causal propagators, $q_{i_{1}, 0}^{(+)}-q_{i_{2}, 0}^{(+)}+\ldots-q_{i_{r}, 0}^{(+)}$, and correspond to spurious singularities, whose dependence is completely dropped in the full sum of contributions (see ref. [60, figure 1]).

The structure of $\lambda_{1}^{ \pm}$exactly corresponds to having all internal lines aligned in the same direction,

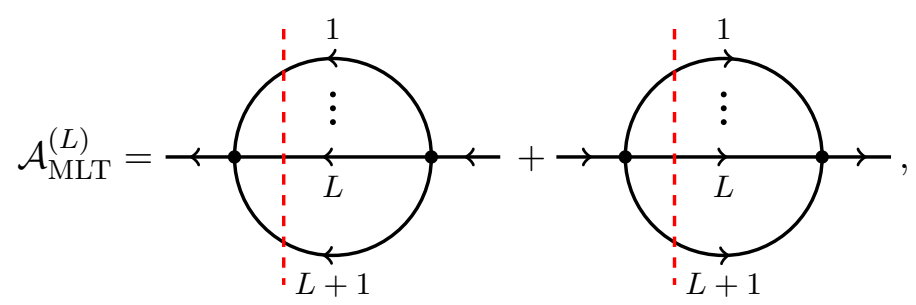

where the two possible directions of the internal lines depend on the flow of the external momentum. Hence, assuming that the two-point function is characterised by only one independent external momentum, $p_{1}$, one has two contributions, when the energy of the incoming particle is positive, $\lambda_{1}^{+}$, or negative, $\lambda_{1}^{-}$. The former and the latter contributions are understood, respectively, in eq. (2.9). Notice that due to the way how $q_{i, 0}^{(+)}$, s have been defined, within LTD, they are always positive quantities. Therefore, the appearance of thresholds comes only from the structure of the energy of the external momenta,

$$
\begin{array}{ll}
\lambda_{1}^{+} \rightarrow \text { threshold if } p_{1,0}>0: & q_{(1, \ldots, L+1)}^{(+)}=p_{1,0}, \\
\lambda_{1}^{-} \rightarrow \text { threshold if } p_{1,0}<0: & q_{(1, \ldots, L+1)}^{(+)}=-p_{1,0} .
\end{array}
$$

Let us emphasise that the representation of eq. (2.9) is valid at all loop orders and it is obtained by the nested application of the Cauchy residue theorem, as discussed in the former section.

To obtain expression (2.9), we parametrise, without loss of generality, all internal lines of an $L$-loop MLT as $q_{i}=\ell_{i}$, with $i=1, \ldots, L$, and $q_{L+1}=-\sum_{i=1}^{L} \ell_{i}-p_{1,0}$. Therefore, in view of this pattern that is supported by factorisation and convolution identities, we define an edge as a set of internal lines, in which all elements connect two vertices,

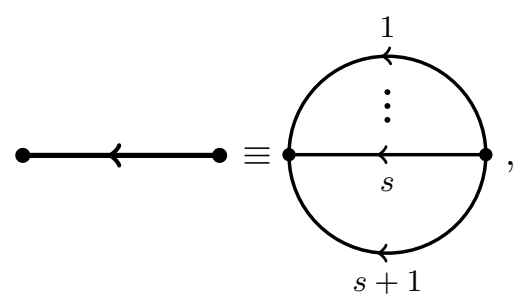


where the thick line in the l.h.s. corresponds to the collection of $s+1$ internal lines aligned in the same direction when external momenta are attached to both vertices. We remark that, independently of the number of internal lines (set elements), momentum conservation has to be satisfied in each vertex.

\section{Causal representation of Feynman integrands}

In ref. [60], it was studied the causal structure of topologies with up to four vertices, Nextto Maximal (NMLT) and Next-to-Next-to Maximal $\left(\mathrm{N}^{2} \mathrm{MLT}\right)$ loop topologies, where a definition and identification of entangled causal propagators were provided. In this section, we recap the results for the NMLT and $\mathrm{N}^{2} \mathrm{MLT}$ configurations and establish two operations, removing and collapsing, to relate topologies with different number of edges and vertices. In fact, as shall be described in the next sections, the more knowledge we have on an $\mathrm{N}^{k}$ MLT configuration, the more applications we can find out of it.

\subsection{Next-to Maximal and Next-to-Next-to Maximal loop topologies}

Following the notation of eq. (2.12), we construct a topology made of three vertices,

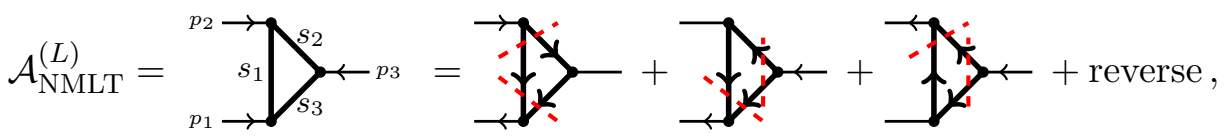

where "reverse" corresponds to changing the direction flow in the internal and external lines. Additionally, as in the MLT configuration (2.10), the red dashed lines that cut internal edges correspond to a pictorial representation of causal propagators. The main difference of this topology, as well as others with more than two vertices, w.r.t. the MLT configuration is the presence of entangled causal thresholds. For instance, each diagram in the r.h.s. of eq. (3.1) contains simultaneously two causal propagators, making its causal representation a bit more involved,

$$
\begin{aligned}
\mathcal{A}_{\mathrm{NMLT}}^{(L)} & =\int_{\ell_{1}, \ldots, \ell_{L}} \frac{1}{x_{L+2}}\left(\frac{1}{\lambda_{1}^{+} \lambda_{2}^{-}}+\frac{1}{\lambda_{1}^{+} \lambda_{3}^{-}}+\frac{1}{\lambda_{2}^{+} \lambda_{3}^{-}}+\left(\lambda_{i}^{+} \leftrightarrow \lambda_{i}^{-}\right)\right) \\
& =\int_{\ell_{1}, \ldots, \ell_{L}} \frac{1}{x_{L+2}} \sum_{\substack{i, j=1 \\
j \neq i}}^{3} \frac{1}{\lambda_{i}^{+} \lambda_{j}^{-}} .
\end{aligned}
$$

The pictorial representation (3.1) or, equivalently, the expression of eq. (3.2) is obtained from the application of LTD, with no loss of generality, at one loop, clearly yielding to the known parametric form of NMLT. Let us remark that this approach is different from the one in [60], where the use of the application of LTD is carried out at three loops. The causal propagators $\lambda_{i}^{ \pm}$of this topology are expressed as follows,

$$
\lambda_{1}^{ \pm}=q_{(1,3), 0}^{(+)} \pm p_{1,0}, \quad \lambda_{2}^{ \pm}=q_{(1,2), 0}^{(+)} \pm p_{2,0}, \quad \lambda_{3}^{ \pm}=q_{(2,3), 0}^{(+)} \pm p_{3,0},
$$

where, as mentioned in the former section, $q_{s_{i}, 0}^{(+)}=\sum_{i \in s_{i}} q_{i, 0}^{(+)}$, contains all internal lines that connects two vertices. In effect, the causal propagators of eq. (3.23) of ref. [60] can 
be extracted by considering the sets $s_{1}=\{1,2\}, s_{2}=\{12\}$ and $s_{3}=\{3, \ldots, L+1\} .{ }^{2}$ Also, we make explicit the dependence on the three external momenta, which are related by momentum conservation $p_{1}+p_{2}+p_{3}=0$.

Let us now draw the attention to a topology built from four vertices, which corresponds to the $\mathrm{N}^{2} \mathrm{MLT}$ configuration. By connecting all vertices themselves, one finds,

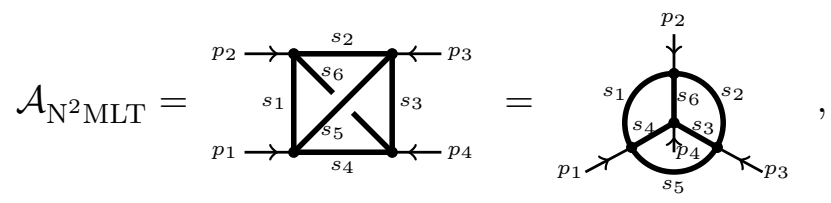

where the r.h.s. clearly is the "planarised" form of the l.h.s. and this topology is often called Mercedes-Benz-like diagram. The causal representation of the $\mathrm{N}^{2} \mathrm{MLT}$ configuration is found to be,

$$
\mathcal{A}_{\mathrm{N}^{2} \mathrm{MLT}}=-\int_{\ell_{1}, \ldots, \ell_{L}} \frac{1}{x_{L+3}}\left[L_{12}^{+} \Lambda_{34}^{-}+L_{13}^{+} \Lambda_{24}^{-}+L_{23}^{+} \Lambda_{14}^{-}+\left(\lambda_{i}^{+} \leftrightarrow \lambda_{i}^{-}\right)\right],
$$

with the shorthand notation,

$$
\Lambda_{i j}^{ \pm}=\left(\frac{1}{\lambda_{i}^{ \pm}}+\frac{1}{\lambda_{j}^{ \pm}}\right), \quad L_{i j}^{ \pm}=\frac{1}{\lambda_{i j}^{ \pm}}\left(\frac{1}{\lambda_{i}^{ \pm}}+\frac{1}{\lambda_{j}^{ \pm}}\right) .
$$

We also suggestively name the causal propagators $\lambda_{i}$ and $\lambda_{i j}$, according to their dependence on the external momenta, $p_{i}$ and $p_{i j}=p_{i}+p_{j}$, respectively,

$$
\begin{array}{ll}
\lambda_{1}^{ \pm}=q_{(1,4,5), 0}^{(+)} \pm p_{1,0}, & \\
\lambda_{2}^{ \pm}=q_{(1,2,6), 0}^{(+)} \pm p_{2,0}, & \lambda_{12}^{ \pm}=q_{(2,4,5,6), 0}^{(+)} \pm p_{12,0}, \\
\lambda_{3}^{ \pm}=q_{(2,3,5), 0}^{(+)} \pm p_{3,0}, & \lambda_{13}^{ \pm}=q_{(1,2,3,4), 0}^{(+)} \pm p_{13,0}, \\
\lambda_{4}^{ \pm}=q_{(3,4,6), 0}^{(+)} \pm p_{4,0}, & \lambda_{23}^{ \pm}=q_{(1,3,5,6), 0}^{(+)} \pm p_{23,0},
\end{array}
$$

with the momentum conservation $p_{1}+p_{2}+p_{3}+p_{4}=0$. Allowing, in this way, to express $\mathcal{A}_{\mathrm{N}^{2} \mathrm{MLT}}$ in the most compact form,

$$
\mathcal{A}_{\mathrm{N}^{2} \mathrm{MLT}}=-\int_{\ell_{1}, \ldots, \ell_{L}} \frac{1}{x_{L+3}} \sum_{\substack{i=1 \\ j=i+1}}^{4} \sum_{\substack{k=1 \\ k \neq i, j}}^{4} L_{i j}^{+} \frac{1}{\lambda_{k}^{-}} .
$$

In order to read this sum, one has to take into account that, because of momentum conservation,

$$
\lambda_{34}^{ \pm}=\lambda_{12}^{\mp}, \quad \lambda_{14}^{ \pm}=\lambda_{23}^{\mp}, \quad \lambda_{24}^{ \pm}=\lambda_{13}^{\mp} .
$$

The expression (3.5) is obtained by applying the LTD theorem on the three-loop Mercedes-Benz like diagram, whose internal lines are expressed by convenience as,

$$
\left\{q_{1}, \ldots, q_{6}\right\}=\left\{\ell_{1}, \ell_{2},-\ell_{2}+\ell_{3}-p_{3}, \ell_{1}-\ell_{3}-p_{1}, \ell_{3},-\ell_{1}+\ell_{2}-p_{2}\right\} .
$$

\footnotetext{
${ }^{2}$ We closely follow the notation of ref. [60], where $i \equiv \ell_{i}, 12 \equiv-\ell_{1}-\ell_{2}+p_{2}$ and $L+1 \equiv-\sum_{i=1}^{L} \ell_{i}-p_{1}$.
} 
Let us remark that although we apply LTD on this topology with six internal lines, the very same behaviour, independently of the loop order, is identical when each edge contains more than a single element.

The notation $\lambda_{i}^{ \pm}$in eq. (3.7) is slightly different to the one found in ref. [60], which is due to the way how the internal sets $s_{i}$ have been constructed. In particular, the extension to $L$ loops in the latter was only carried out in the set $s_{5}$, by considering $s_{5}=\{4, \ldots, L+1\}$, while each of the other sets contains only one element. Hence, the notation,

$$
q_{\left(i_{1}, i_{2}, \ldots, i_{r}\right), 0}^{(+)}=\sum_{k \in i_{1} \cup i_{2} \cup \cdots \cup i_{r}} q_{k, 0}^{(+)}
$$

is straightforwardly extended.

\subsection{Removing and collapsing procedures}

The causal representation of $\mathrm{N}^{2} \mathrm{MLT}$ displays a much richer structure than the NMLT configuration. It is for this reason that the question about the existence of a relation among these loop topologies, and extension to others with higher orders in edges and vertices, is well posed. Then, to understand this pattern, we give a preliminary answer to the latter by introducing two procedures at the level of the topologies: removing and collapsing.

\subsubsection{Removing}

In order to start this discussion, let us recap the way how the causal representation of the NMLT and $\mathrm{N}^{2}$ MLT configurations have been generated. In particular, the NMLT configuration was obtained through the application of LTD on a one-loop three-point scalar integral, whereas the $\mathrm{N}^{2}$ MLT configuration was generated by the application of LTD on a three-loop three-point scalar integral. However, the very same structure of both integrands holds when more internal lines are included and it is the reason for working with edges in the present manuscript. Notice that the only difference when considering more internal lines (or more elements in the edges) is the presence of additional $q_{i, 0}^{(+)}$'s in the definition of $\lambda_{i}^{ \pm}$. Hence, understanding the removing operation of elements in an edge is straightforward: one sets the corresponding $q_{i, 0}^{(+)} \rightarrow 0$. Interestingly, this operation can also directly be applied on edges, taking into account that the resulting topology, after the removing, cannot be disconnected.

Then, with this procedure, we consider the $\mathrm{N}^{2} \mathrm{MLT}$ topology and remove, with no loss of generality, the edges $s_{5}$ and $s_{6}$ (see eq. (3.4)). This procedure corresponds to setting $q_{s_{5}, 0}^{(+)}, q_{s_{6}, 0}^{(+)} \rightarrow 0$ in the definition of $\lambda_{i}^{ \pm}$'s,

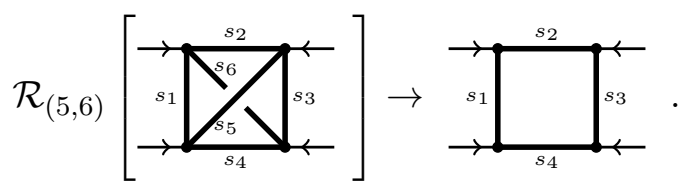

Here and in the following, the operator $\mathcal{R}_{i_{1}, i_{2}, \ldots, i_{N}}[\#]$ corresponds to removing the edges $s_{i_{1}}, s_{i_{2}}, \ldots, s_{i_{N}}$ in the multi-loop topology. In effect, this operation introduces simplifica- 
tions in the expression (3.5),

$$
L_{13}^{ \pm} \Lambda_{24}^{\mp} \rightarrow \frac{1}{\lambda_{1}^{ \pm} \lambda_{3}^{ \pm}} \Lambda_{24}^{\mp}=\frac{1}{\lambda_{2}^{\mp} \lambda_{4}^{\mp}} \Lambda_{13}^{ \pm} .
$$

Thus, recovering the causal representation of a one-loop box calculated from the standard application of LTD, with only 6 causal thresholds. A noteworthy feature of this operation is that the dependence on the causal threshold $\lambda_{13}^{ \pm}$is completely dropped, which is in agreement with the diagrammatic perspective, since no edge is connecting the external momenta $p_{1}$ and $p_{3}$ (or $p_{2}$ and $p_{4}$ ). Nonetheless, the same simplification is obtained by removing either $s_{5}$ or $s_{6}$ as is noted in the first and second term in the r.h.s. of eq. (3.13), respectively.

In the former result, we were interested in the part of the integrand that only contains $\lambda_{i}^{ \pm}$. However, setting any $q_{s_{i}, 0}^{(+)} \rightarrow 0$ introduces a singularity in $x_{L+3}$, which is healed by taking the following limit,

$$
d \mathcal{A}_{\mathrm{N}^{2} \mathrm{MLT}}^{\left(L-\left|s_{i}\right|\right)}=\lim _{q_{s_{i}}^{(+)} \rightarrow 0} 2 q_{s_{i}}^{(+)} d \mathcal{A}_{\mathrm{N}^{2} \mathrm{MLT}}^{(L)}
$$

where the integrand $d \mathcal{A}_{N}^{(L)}$ has previously defined in eq. (2.2) and, abusing of notation, $\left|s_{i}\right|$ corresponds to the number of elements of the set $s_{i}$. In case of removing more internal lines, one elaborates on the application of a nested limit.

So far we have considered topologies constructed from linear Feynman propagators, however, the removing procedure (3.14) can be extended to the general case with raised powers in the Feynman propagators. For instance, removing one internal line, say $i$, whose propagator is raised to power $m_{i}$, one gets,

$$
d \mathcal{A}_{\mathrm{N}^{k} \mathrm{MLT}}^{(L-1)}=\frac{(-1)^{m_{i}-1}}{2^{m_{i}-1}\left(2 m_{i}-3\right) ! !} \lim \left(2 q_{i}^{(+)}\right)^{2 m_{i}-1} d \mathcal{A}_{\mathrm{N}^{k} \mathrm{MLT}}^{(L)},
$$

that nestedly can be applied to all internal lines of a given edge.

\subsubsection{Collapsing}

In the former discussion, we provided a procedure to obtain, without the application of the LTD formalism, topologies that are related by the number of vertices and regardless of the number of edges. It is indeed desirable to recover topologies with lower number of vertices by applying a similar procedure like removing. The motivation for doing this is to compute one complicated topology once and for all and, as a byproduct, all topologies with lower number of vertices. To this end, in this section, we introduce the collapsing procedure that, together with removing, allows for a unified framework to generate any causal representation from the richest topology with the highest number of vertices and edges. Let us emphasise that the collapsing procedure is not intended to be an operation that shrinks an edge to a point. On the contrary, every time this operation is applied, it removes one vertex and the edges that are attached to it. Thus, passing at each iteration from $n$ to $n-1$ vertices. 
In order to elaborate on the collapsing procedure, we consider the $\mathrm{N}^{2}$ MLT configuration and remove the edges $s_{3}, s_{4}, s_{6}$ and the external momenta $p_{4}$ (see eq. (3.4)),

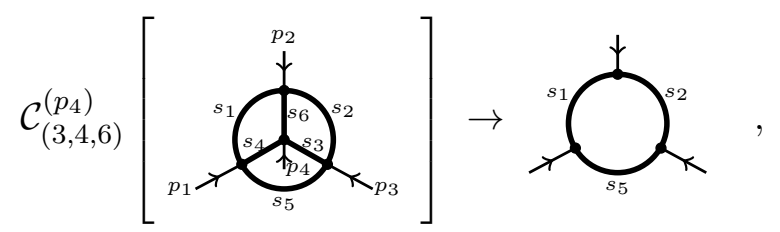

with $\mathcal{C}_{\left(i_{1}, i_{2}, \ldots, i_{N}\right)}^{\left(p_{r_{1}}\right)}[\#]$ the operator that collapses the internal lines $i_{1}, i_{2}, \ldots, i_{N}$ and removes the external momenta $p_{r_{1}}$. Certainly, the r.h.s. of eq. (3.16) should recover the known structure for NMLT. However, when removing these edges together with the external momentum, one notices that there is a causal propagator that introduces a singularity, $\lambda_{4}^{ \pm}$. Hence, to make a smooth transition from $\mathrm{N}^{2} \mathrm{MLT}$ to NMLT, we firstly compute the limit when $\lambda_{4}^{ \pm} \rightarrow 0$ and nestedly the ones that involve, in this case, $s_{3}, s_{4}, s_{6}$ and $p_{4,0}$,

$$
d \mathcal{A}_{\mathrm{NMLT}}=-\frac{1}{2} \lim _{s_{3}, s_{4}, s_{6} \rightarrow 0} \prod_{i \in s_{3} \cup s_{4} \cup s_{6}} 2 q_{i, 0}^{(+)} \lim _{\lambda_{5}^{ \pm} \rightarrow 0} \lambda_{4}^{ \pm} d \mathcal{A}_{\mathrm{N}^{2} \mathrm{MLT}} \cdot
$$

The nested limits in eq. (3.17) express all $\lambda_{i j}^{ \pm}$as,

$$
\lambda_{12}^{ \pm} \rightarrow \lambda_{3}^{\mp}, \quad \lambda_{13}^{ \pm} \rightarrow \lambda_{2}^{\mp}, \quad \lambda_{23}^{ \pm} \rightarrow \lambda_{1}^{\mp},
$$

where the momentum conservation $p_{1}+p_{2}+p_{3}=0$ is taken into account, thus, recovering the very same expression of eq. (3.2).

Analogously, for the $\mathcal{A}_{\mathrm{MLT}}$, we have a nested limit,

$$
d \mathcal{A}_{\mathrm{MLT}}=\frac{1}{4} \lim _{s_{2}, \ldots, s_{6} \rightarrow 0} \prod_{i \in s_{2} \cup \ldots \cup s_{6}} 2 q_{i, 0}^{(+)} \lim _{\lambda_{3}^{ \pm} \rightarrow 0} \lambda_{3}^{ \pm} \lim _{\lambda_{6}^{ \pm} \rightarrow 0} \lambda_{4}^{ \pm} d \mathcal{A}_{\mathrm{N}^{2} \mathrm{MLT}}
$$

where $\lambda_{2}^{\mp} \rightarrow \lambda_{1}^{ \pm}$and $p_{1}+p_{2}=0$.

In the former discussions, we have introduced two procedures, removing and collapsing, to generate any kind of loop topology independently of the number of vertices and edges. In particular, we consider the relations between $\mathrm{N}^{2} \mathrm{MLT}$ with less number of edges and the transition from $\mathrm{N}^{2} \mathrm{MLT}$ to NMLT and MLT. We observe that starting from an $\mathrm{N}^{k} \mathrm{MLT}$ topology, with $k+2$ vertices, allows for a straightforward reconstruction of the causal representation of all lower topologies. It is also clear that more complexity is expected when more vertices are included. However, as shall be noted in the following section, one can use the removing and collapsing procedures to understand the causal representation of $\mathrm{N}^{k}$ MLT from $\mathrm{N}^{k-1}$ MLT configurations.

The most general topology with a given number of vertices is generated by considering all edges, namely, all possible connections among vertices, as done in NMLT and $\mathrm{N}^{2}$ MLT. The reason of being the most general as possible in the generation of topologies is to extract without any ambiguity the causal propagators that appear in a given topology. The aim of the latter is to generate yet a causal representation of multi-loop topologies without making use of the LTD formalism, by means of the application of the nested residue. 

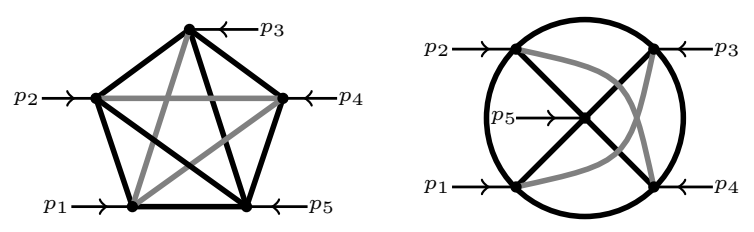

Figure 1. Multi-loop topology with five vertices and ten internal lines.

\section{Five-vertex topologies and beyond}

Following the same discussion of the former section, we start from a topology containing five vertices with all possible adjacencies among themselves, as depicted in figure 1, where the grey lines do not cross among themselves.

\subsection{Causal representation of five-vertex topologies}

In order to harness of the LTD formalism and, therefore, the causal representation, we need to consider a topology at six loops with ten denominators,

$$
q_{i}= \begin{cases}q_{i}=\ell_{i}, & i=1, \ldots, 6 \\ \ell_{1}-\ell_{4}-\ell_{5}-p_{1}, & i=7 \\ \ell_{2}-\ell_{1}-\ell_{6}-p_{2}, & i=8 \\ \ell_{3}-\ell_{2}+\ell_{5}-p_{3}, & i=9 \\ \ell_{4}-\ell_{3}+\ell_{6}-p_{4} & i=10\end{cases}
$$

whose causal propagators are found to be,

$$
\begin{array}{lll}
\lambda_{1}^{ \pm}=q_{(1,4,5,7), 0}^{(+)} \pm p_{1,0}, & \lambda_{12}^{ \pm}=q_{(2,4,5,6,7,8), 0}^{(+)} \pm p_{12,0}, & \lambda_{24}^{ \pm}=q_{(1,2,3,4,8,10), 0}^{(+)} \pm p_{24,0}, \\
\lambda_{2}^{ \pm}=q_{(1,2,6,8), 0}^{(+)} \pm p_{2,0}, & \lambda_{13}^{ \pm}=q_{(1,2,3,4,7,9), 0}^{(+)} \pm p_{13,0}, & \lambda_{35}^{ \pm}=q_{(2,3,5,7,8,10), 0}^{(+)} \pm p_{35,0}, \\
\lambda_{3}^{ \pm}=q_{(2,3,5,9), 0}^{(+)} \pm p_{3,0}, & \lambda_{23}^{ \pm}=q_{(1,3,5,6,8,9), 0}^{(+)} \pm p_{23,0}, & \lambda_{34}^{ \pm}=q_{(2,4,5,6,9,10), 0}^{(+)} \pm p_{34,0}, \\
\lambda_{4}^{ \pm}=q_{(3,4,6,10), 0}^{(+)} \pm p_{4,0}, & \lambda_{45}^{ \pm}=q_{(3,4,6,7,8,9), 0}^{(+)} \pm p_{45,0}, & \lambda_{25}^{ \pm}=q_{(1,2,6,7,9,10), 0}^{(+)} \pm p_{25,0}, \\
\lambda_{5}^{ \pm}=q_{(7,8,9,10), 0}^{(+)} \pm p_{5,0}, & \lambda_{14}^{ \pm}=q_{(1,3,5,6,7,10), 0}^{(+)} \pm p_{14,0}, & \lambda_{15}^{ \pm}=q_{(1,4,5,8,9,10), 0}^{(+)} \pm p_{15,0} .
\end{array}
$$

Let us remark that the causal representation of this diagram will contain a product of four causal propagators, which is expected e.g. from a one-loop pentagon - no internal lines in figure 1. Nevertheless, not all products of $\lambda_{i}$ 's in (4.2) will appear in the representation. In effect, there will be products of two $\lambda_{i}^{ \pm}$'s, that cannot appear because of the incompatible alignment of internal and external momenta. In other words, these two causal propagators will correspond, from a graphical point of view, to an overlapping. Let us illustrate this point by considering a possible entanglement between $\lambda_{12}^{ \pm}, \lambda_{14}^{ \pm}$and $\lambda_{12}^{ \pm}, \lambda_{34}^{ \pm}$. In figure 2 (left), we note that the causal thresholds $\lambda_{12}^{ \pm}, \lambda_{14}^{ \pm}$cannot be considered together when providing a candidate for the causal representation, whereas, $\lambda_{12}^{ \pm}, \lambda_{34}^{ \pm}$, in figure 2 (right), 

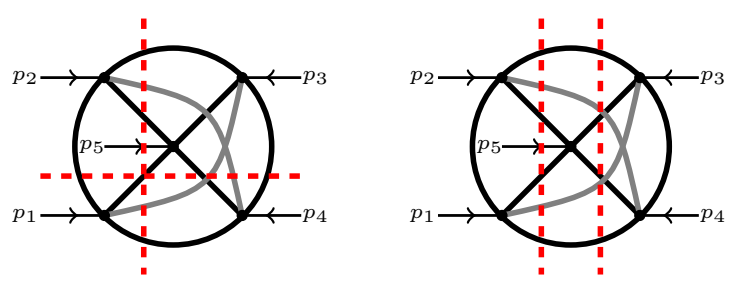

Figure 2. Loop topologies with (left:) two overlapped and (right:) two entangled thresholds.

appear with combinations of other $\lambda_{i}^{ \pm}$'s. Similarly, we can apply the same procedure to tuples of length three. ${ }^{3}$

Remarkably, the features described for the loop topology of figure 2 with either overlapped or entangled causal thresholds may be related to the Steinmann relations [70-76], since they state that double discontinuities in overlapping cuts vanish. Hence, from the latter there is the support that $\lambda_{12}^{ \pm}$and $\lambda_{14}^{ \pm}$cannot appear together in the causal representation of this loop topology. The Steinmann relations have been considered in recent works [77-81] and read as follows,

$$
\operatorname{Disc}_{s_{I}}\left(\operatorname{Disc}_{s_{J}} \mathcal{A}_{N}^{(L)}\right)=0, \quad \text { where }\left\{\begin{array}{l}
I \not \subset J \\
J \not \subset I
\end{array},\right.
$$

where $\operatorname{Disc}_{s_{I}} \mathcal{A}_{N}^{(L)}$ represents the discontinuity of the scattering amplitude (or Feynman integral) of eq. (2.1) at the kinematic invariant $s_{I}=\left(\sum_{i \in I} p_{i}\right)^{2}$ generated by the external momenta that belong to the set $I$. A naive interpretation of the Steinmann relations within our framework may be given by promoting the kinematic invariants $s_{I}$ to the sum of energies of external momenta present in the causal propagators.

In effect, with the above considerations, one can provide an Ansatz, which, in principle, has to be the most general one. Additionally, due to the constraints given by the tuples of $\lambda_{i}^{ \pm}$, one can significantly reduce the number of parameters to fit or, equivalently, the number of linear equations to solve. In order to speed up their solution, we make use of the analytic reconstruction over finite fields [82-85]. In particular, with the use of the publicly C++ code FiniteFlow [84]. Hence, the causal representation of the six-loop five-point scalar integral becomes,

$$
\mathcal{A}_{\mathrm{N}^{3} \mathrm{MLT}}^{(L)}=\int_{\ell_{1}, \ldots, \ell_{L}} \frac{1}{x_{L+4}} \sum_{\substack{i=1 \\ j=i+1}}^{5} \sum_{\substack{k=1 \\ l=k+1 \\ k, l \neq i, j}}^{5} L_{i j}^{+} L_{k l}^{-} .
$$

We remark that this expression has been obtained from an Ansatz and numerically checked with the residues generated from the nested application of LTD. Moreover, we can perform further checks by considering the removing and collapsing procedures, presented in section 3 .

\footnotetext{
${ }^{3}$ This procedure has been implemented in MAтнEмAтіcA by studying the direction of all causal propagators according to the presence of external momenta.
} 
Since the expression (4.4) corresponds to $\mathrm{N}^{3} \mathrm{MLT}$, we can begin collapsing all edges that connect the momentum $p_{5}$ with other vertices, by setting to zero the edges $s_{7}, s_{8}, s_{9}, s_{10}$ and the external momentum $p_{5}$. This operation leads to $\lambda_{5}^{ \pm} \rightarrow 0$. Then, looking only at the contribution proportional $\left(\lambda_{5}^{ \pm}\right)^{-1}$ in (4.4),

$$
\mathcal{A}_{\mathrm{N}^{3} \mathrm{MLT}}^{(L)}=\int_{\ell_{1}, \ldots, \ell_{L}} \frac{1}{x_{L+4}}\left(\frac{1}{\lambda_{5}^{-}} \sum_{\substack{i=1 \\ j=i+1}}^{4} \sum_{\substack{k=1 \\ k \neq i, j}}^{4} L_{i j}^{+} \frac{1}{\lambda_{k 5}^{-}}+\left(\lambda_{i}^{+} \leftrightarrow \lambda_{j}^{+}\right)\right)+\mathcal{O}\left(\left(\lambda_{5}^{ \pm}\right)^{0}\right),
$$

and applying the collapsing procedure of eq. (3.17), one exactly recovers the known result of eq. (3.5), after setting the corresponding $q_{s_{i}} \rightarrow 0$ in the definition of the causal propagators (4.2) or equivalently considering that $\lambda_{k 5}^{ \pm} \rightarrow \lambda_{k}^{ \pm}$.

In ref. [61], it has been recently computed the multi-loop LTD decomposition of a scattering amplitude at four loops. On top of it, a causal representation of the four-loop topology, with five external momenta and eight internal lines, was also presented, which is expressed in terms of thirteen causal propagators. In fact, the topology we are referring to corresponds to the diagram of figure 1 without grey lines. Furthermore, within the removing procedure presented in section 3 , we obtain the causal representation by simply setting to zero the lines $q_{s_{5}}$ and $q_{s_{6}}$. By doing so, and looking at the definition of causal propagators (4.2), we note that, in principle, we get more $\lambda_{i}^{ \pm}$than expected. Also, the expression in eq. (4.4) contains 120 terms instead of 98, as in the four-loop diagram. Nonetheless, the same pattern, already seen in eq. (3.13) for the transition of a three-loop Mercedes-Benz like diagram to a one-loop box, is found here. In particular,

$$
L_{13}^{ \pm} \rightarrow \frac{1}{\lambda_{1}^{ \pm} \lambda_{3}^{ \pm}}, \quad L_{24}^{ \pm} \rightarrow \frac{1}{\lambda_{2}^{ \pm} \lambda_{4}^{ \pm}},
$$

dropping the dependence, in the causal representation, on $\lambda_{13}^{ \pm}$and $\lambda_{24}^{ \pm}$, and, thus, recovering the 98 terms. $^{4}$

An alternative explanation to this cancellation, directly from the diagram of figure 1 , can be given by noticing that the external momenta $p_{1}, p_{3}$ or, equivalently, $p_{2}, p_{4}$ are not connected among themselves. Hence, no threshold singularity may be generated from these external momenta.

Therefore, based on the above-mentioned considerations, we remark that the application of a four-loop five-point scalar diagram, with eight internal lines, studied in ref. [61] is a particular case of the $\mathcal{A}_{\mathrm{N}^{3} \mathrm{MLT}}^{(L)}$ presented in this section. We also observe that the former cannot reconstruct the full structure of $\mathcal{A}_{\mathrm{N}^{2} \mathrm{MLT}}^{(L)}$, by using the collapsing procedure. It, however, can reconstruct the causal representation of a one-loop box, by removing all internal lines that connect to e.g. $p_{5}$. On top of it, by applying the removing and collapsing procedures on $\mathcal{A}_{\mathrm{N}^{3} \mathrm{MLT}}^{(L)}$, we generate the causal representation of topologies of up-to four loops. These topologies are summarised in table 1 , where $\operatorname{dim} \lambda_{i}^{ \pm}$, as shall be explained in the following section, corresponds to the number of causal propagators present

\footnotetext{
${ }^{4}$ Let us also comment that for the topology with five vertices and eight edges, we have experimented an alternative approach based on the partial fractioning through the automated codes MulTIVARIATEAPART [86] and the Singular [87] library pfd.lib [88], finding 102 causal terms instead of 98.
} 


\begin{tabular}{|c|c|c|c|}
\hline Topology & $\operatorname{dim} \lambda_{i}^{ \pm}$ & Entanglement & \# Causal terms \\
\hline & 1 & 1 & 2 \\
\hline & 3 & 2 & 6 \\
\hline & 6 & 3 & 20 \\
\hline & 1 & 1 & 2 \\
\hline & 6 & 3 & 20 \\
\hline & 10 & 3 & 70 \\
\hline & 11 & 3 & 76 \\
\hline & 6 & 3 & 20 \\
\hline & 7 & 3 & 24 \\
\hline & 13 & 4 & 98 \\
\hline
\end{tabular}

Table 1. Selected topologies generated from the causal representation of the $\mathrm{N}^{3} \mathrm{MLT}$ configuration by applying the removing and collapsing procedures.

in a given topology. Entanglement accounts for the number of causal propagators present in each causal term. \# Causal terms corresponds to the number of terms expressed in terms of causal propagators. For instance, the one-loop box discussed in section 3.2.1 has $\operatorname{dim} \lambda_{i}^{ \pm}=6$ (causal thresholds), Entanglenment $=3$ and \# Causal terms $=20$.

\subsection{Causal propagators beyond five vertices}

In the former sections, we study the causal representation of $\mathcal{A}_{\mathrm{N}^{k} \mathrm{MLT}}^{(L)}$, with $k=0,1,2,3$, finding an interesting pattern in the structure of $\lambda_{i}^{ \pm}$. In the following, we summarise our findings and generalise to the $n$-vertex case. 
(i) A topology with $n$ vertices, containing $n$ external momenta, has

$$
\operatorname{dim} \lambda_{i}^{ \pm}=2^{n-1}-1
$$

with $\operatorname{dim} \lambda_{i}^{ \pm}$the number of causal propagators presented in each topology. In the results of eqs. (3.3), (3.7) and (4.2), we use momentum conservation to have the first $n$ causal propagators in terms of a single external momentum. Namely, that the largest subset of external momenta, $\left\{p_{1}, p_{2}, \ldots, p_{n-1}\right\}$, is replaced by a subset with only one element, $\left\{p_{n}\right\}$.

Let us remark that the numbers in eq. (4.7) accounts for the causal propagators when all possible connections among vertices are taken into account. Hence, if there are tuples of vertices that are not connected, they have to be subtracted out from the initial counting. This is indeed the case of the five-vertex diagram of figure 1 , with and without the grey edges, since in the latter the external momenta $p_{2}, p_{4}$ and $p_{1}, p_{3}$ are not connected. Therefore, these subsets have to be removed from (4.7), as found in the previous section by considering the direct application of LTD.

(ii) The structure of each causal propagator is straightforwardly obtained, provided that all subsets of external momenta are properly generated. As mention in (i), we make use of momentum conservation to have subsets of momenta with the minimal number of elements. Thus, for a topology with $n$ external momenta, we consider subsets with up to $[n / 2]$ elements, ${ }^{5}$ allowing for a straightforward generation of all causal propagators.

For subsets with only one element, e.g. $p_{i}$, we only need to consider the internal lines that are connecting the momentum $p_{i}$. Similarly, for subsets with more than one element, say $p_{i}$ and $p_{j}$, we collapse these momenta in a single one, $p_{i j}$, and consider all the internal lines that are connecting to this "new" external momentum, taking into account that the connecting line between $p_{i}$ and $p_{j}$ is not considered.

Notably, from (i), we can know all features of the most general topology made of $n$ vertices, because the maximum number of edges, loops and causal propagators are directly related to the number of vertices, as it is summarised in table 2 for all topologies studied until now as well as the generalisation to $n$ vertices.

In order to illustrate these points, let us consider, for illustrative reasons, a two-loop planar double box with six external legs (see figure 3). Hence, according to (i), one starts with 31 causal propagators, nevertheless, only 15 true ones correspond to the possible threshold singularities that can be obtained in a topology of seven propagators. Thus,

\footnotetext{
${ }^{5}[x]$ corresponds to the integral part or integer part of $x$.
} 


\begin{tabular}{|c|c|c|c|c|}
\hline & vertices & Edges & Loops & $\operatorname{dim} \lambda_{i}^{ \pm}$ \\
\hline & 2 & 1 & 0 & 1 \\
\hline$\vdots$ & 3 & 3 & 1 & 3 \\
\hline$\vdots$ & 4 & 6 & 3 & 7 \\
\hline$\vdots$ & $n$ & 10 & 6 & 15 \\
\hline$\vdots$ & $n$ & $\vdots$ & $\vdots$ & $\vdots$ \\
\hline
\end{tabular}

Table 2. Features of topologies with Maximal number of edges, loops and causal propagators according to the number of vertices. In the topology with $n$ vertices, the grey vertices account for all possible connections among vertices.
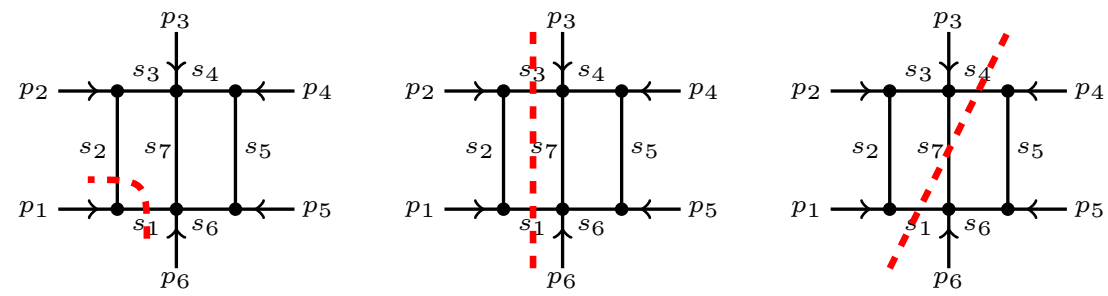

Figure 3. Particular two-loop topology with six vertices and seven internal lines.

taking into account (ii), we find,

$$
\begin{array}{lll}
\lambda_{1}^{ \pm}=q_{(1,2)}^{(+)} \pm p_{1,0}, & \lambda_{6}^{ \pm}=q_{(1,6,7)}^{(+)} \pm p_{6,0}, & \lambda_{45}^{ \pm}=q_{(4,6)}^{(+)} \pm p_{45,0}, \\
\lambda_{2}^{ \pm}=q_{(2,3)}^{(+)} \pm p_{2,0}, & \lambda_{12}^{ \pm}=q_{(1,3)}^{(+)} \pm p_{12,0}, & \lambda_{56}^{ \pm}=q_{(1,5,7)}^{(+)} \pm p_{56,0}, \\
\lambda_{3}^{ \pm}=q_{(3,4,7)}^{(+)} \pm p_{3,0}, & \lambda_{16}^{ \pm}=q_{(2,6,7)}^{(+)} \pm p_{16,0}, & \lambda_{123}^{ \pm}=q_{(1,4,7)}^{(+)} \pm p_{123,0}, \\
\lambda_{4}^{ \pm}=q_{(4,5)}^{(+)} \pm p_{4,0}, & \lambda_{23}^{ \pm}=q_{(2,4,7)}^{(+)} \pm p_{23,0}, & \lambda_{234}^{ \pm}=q_{(2,5,7)}^{(+)} \pm p_{234,0}, \\
\lambda_{5}^{ \pm}=q_{(5,6)}^{(+)} \pm p_{5,0}, & \lambda_{34}^{ \pm}=q_{(3,5,7)}^{(+)} \pm p_{34,0}, & \lambda_{345}^{ \pm}=q_{(3,6,7)}^{(+)} \pm p_{345,0} .
\end{array}
$$

The structure of all $\lambda_{i}^{ \pm}$in eq. (4.8) are in agreement with the ones found with the standard application of the nested residue implemented in LотTY [89].

\section{Causal representation of loop topologies without LTD}

In sections 3 and 4, we study the causal representation of loop topologies made of up-to five vertices, by following the application of LTD. Interestingly, the structure of these 
topologies, thanks to the knowledge of all causal propagators, can be inferred by properly accommodating tuples of $\lambda_{i}^{ \pm}$. This is carried out by following the approaches summarised in figure 2 and the items (i) and (ii). In this section, we generate the causal representation of loop topologies made of up-to nine vertices, without making use of LTD, but considering only the collapsing procedure. The latter is to cross-check the result, obtained from an Ansatz, of an $\mathrm{N}^{k} \mathrm{MLT}$ with the corresponding lower topology, $\mathrm{N}^{k-1} \mathrm{MLT}$.

Since the relevant information of the causal representation of a given topology only comes from the part that contains the causal propagators, $\lambda_{i}^{ \pm}$, we do not consider the prefactors $x_{L+k}$. The latter indeed can be recovered at the end of the calculation.

- The MLT configuration can only have a causal threshold. Hence,

$$
d \mathcal{A}_{\mathrm{MLT}}^{(L)} \sim \frac{1}{\lambda_{1}^{+}}+\frac{1}{\lambda_{1}^{-}}
$$

- The NMLT configuration has 3 causal propagators that do not overlap among themselves. The only subtlety that one has to account for is the direction of $\lambda_{i}^{ \pm}$. Then, by organising these causal propagators in couples,

$$
d \mathcal{A}_{\mathrm{NMLT}}^{(L)} \sim \sum_{\sigma_{i j}} \frac{1}{\lambda_{i}^{+} \lambda_{j}^{-}}
$$

with $\sigma_{i j}=\{\{1,2\},\{1,3\},\{2,1\},\{2,3\},\{3,1\},\{3,2\}\}$.

- The $\mathrm{N}^{2} \mathrm{MLT}$ configuration has 7 causal propagators that working with subsets can be understood as,

$$
\vec{\lambda}_{i}^{ \pm}=\{\{1\},\{2\},\{3\},\{4\},\{1,2\},\{1,3\},\{2,3\}\},
$$

where, abusing of notation, $\vec{\lambda}_{i}^{ \pm}$is an array with all causal propagators. Let us emphasise that we are interested in the structure of the latter given by the external momenta. For this reason, the expression of the edges in terms of $q_{s_{i}, 0}^{(+)}$is not needed. Noticed that two causal propagators that have two external momenta, e.g. $\{1,2\}$ and $\{1,3\}$, cannot be entangled. Thus, we have,

$$
d \mathcal{A}_{\mathrm{N}^{2} \mathrm{MLT}}^{(L)} \sim \sum_{\substack{i=1 \\ j=i+1}}^{4} \frac{1}{\lambda_{i j}^{+}} f_{i j}\left(\left\{\lambda^{ \pm}\right\}\right)
$$

where in the test function $f_{i j}$, we are left with causal propagators with a single external momentum, $\{i\}$. Then, thanks to this notation, it is clear that $\lambda_{i}^{ \pm}$(or $\lambda_{j}^{ \pm}$) can be "entangled" with $\lambda_{i j}^{ \pm}$, thus,

$$
d \mathcal{A}_{\mathrm{N}^{2} \mathrm{MLT}}^{(L)} \sim \sum_{\substack{i=1 \\ j=i+1}}^{4} \frac{1}{\lambda_{i j}^{+}}\left(\frac{1}{\lambda_{i}^{+}}+\frac{1}{\lambda_{j}^{+}}\right) \bar{f}_{i j}\left(\left\{\lambda_{k}^{ \pm}\right\}\right)=\sum_{\substack{i=1 \\ j=i+1}}^{4} L_{i j, i, j}^{+} \bar{f}_{i j}\left(\left\{\lambda_{k}^{ \pm}\right\}\right)
$$


but also to causal propagators whose flow is in the opposite direction, e.g.,

$$
\frac{1}{\lambda_{12}^{+}}\left(\frac{1}{\lambda_{1}^{+}}+\frac{1}{\lambda_{2}^{+}}\right)\left(\frac{1}{\lambda_{3}^{-}}+\frac{1}{\lambda_{4}^{-}}\right) .
$$

This consideration amounts to the results found in eq. (3.8),

$$
d \mathcal{A}_{\mathrm{N}^{2} \mathrm{MLT}}^{(L)} \sim \sum_{\substack{i=1 \\ j=i+1}}^{4} \sum_{\substack{k=1 \\ k \neq i, j}}^{4} L_{i j}^{+} \frac{1}{\lambda_{k}^{-}} .
$$

- Let us now draw our attention to the $\mathrm{N}^{3} \mathrm{MLT}$, whose 15 causal propagators can be chosen to be,

$$
\begin{aligned}
\vec{\lambda}_{i}^{ \pm}= & \{\{1\},\{2\},\{3\},\{4\},\{5\}, \\
& \{1,2\},\{1,3\},\{1,4\},\{1,5\},\{2,3\},\{2,4\},\{2,5\},\{3,4\},\{3,5\},\{4,5\}\} .
\end{aligned}
$$

Differently to the $\mathrm{N}^{2} \mathrm{MLT}$ configuration, there will be products of pairs $\lambda_{i j}^{ \pm}$that will be allowed. In fact,

$$
\begin{aligned}
& \{\{\{1,2\},\{3,4\}\},\{\{1,2\},\{3,5\}\},\{\{1,2\},\{4,5\}\},\{\{1,3\},\{2,4\}\},\{\{1,3\},\{2,5\}\} \\
& \{\{1,3\},\{4,5\}\},\{\{1,4\},\{2,3\}\},\{\{1,4\},\{2,5\}\},\{\{1,4\},\{3,5\}\},\{\{1,5\},\{2,3\}\} \\
& \{\{1,5\},\{2,4\}\},\{\{1,5\},\{3,4\}\},\{\{2,3\},\{4,5\}\},\{\{2,4\},\{3,5\}\},\{\{2,5\},\{3,4\}\}\}
\end{aligned}
$$

where, due to the knowledge of the $\mathrm{N}^{2} \mathrm{MLT}$, each subset can be understood as,

$$
\{\{i, j\},\{k, l\}\} \rightarrow L_{i j}^{+} L_{k l}^{-}+L_{i j}^{-} L_{k l}^{+} .
$$

Thus, recovering the results of eq. (4.4),

$$
d \mathcal{A}_{\mathrm{N}^{3} \mathrm{MLT}}^{(L)} \sim \sum_{\substack{i=1 \\ j=i+1}}^{5} \sum_{\substack{k=1 \\ l=k+1 \\ k, l \neq i, j}}^{5} L_{i j}^{+} L_{k l}^{-}
$$

- The results previously described were computed by means of the application of LTD on topologies of up-to six loops, then the cross-check is straightforward. Let us now consider a more involved topology, the $\mathrm{N}^{4} \mathrm{MLT}$, whose 31 causal propagators can be chosen to be,

$$
\begin{aligned}
\vec{\lambda}_{i}^{ \pm}= & \{\{1\},\{2\},\{3\},\{4\},\{5\},\{6\}, \\
& \{1,2\},\{1,3\},\{1,4\},\{1,5\},\{1,6\},\{2,3\},\{2,4\},\{2,5\},\{2,6\},\{3,4\},\{3,5\}, \\
& \{3,6\},\{4,5\},\{4,6\},\{5,6\},\{1,2,3\},\{1,2,4\},\{1,2,5\},\{1,3,4\},\{1,3,5\}, \\
& \{1,4,5\},\{2,3,4\},\{2,3,5\},\{2,4,5\},\{3,4,5\}\} .
\end{aligned}
$$


Firstly, by studying tuples of three elements, we note that none of them can be entangled together, then,

$$
d \mathcal{A}_{\mathrm{N}^{4} \mathrm{MLT}}^{(L)} \sim \sum_{\substack{i=1 \\ j=i+1 \\ k=j+1}}^{6} \frac{1}{\lambda_{i j k}^{+}} f_{i j k}\left(\left\{\lambda_{r s}\right\},\left\{\lambda_{r}\right\}\right) .
$$

Now considering the product of pairs, similar to (5.9), we find 45 subsets of the form,

$$
\begin{aligned}
& \{\{\{1,2\},\{3,4\}\},\{\{1,2\},\{3,5\}\},\{\{1,2\},\{3,6\}\},\{\{1,2\},\{4,5\}\},\{\{1,2\},\{4,6\}\}, \\
& \{\{1,2\},\{5,6\}\},\{\{1,3\},\{2,4\}\},\{\{1,3\},\{2,5\}\},\{\{1,3\},\{2,6\}\},\{\{1,3\},\{4,5\}\}, \\
& \{\{1,3\},\{4,6\}\},\{\{1,3\},\{5,6\}\},\{\{1,4\},\{2,3\}\},\{\{1,4\},\{2,5\}\},\{\{1,4\},\{2,6\}\}, \\
& \ldots\} .
\end{aligned}
$$

Notice that the intersection of the elements of the subset $\{\{i, j\},\{k, l\}\}$ is empty, $\{i, j\} \cap\{k, l\}=\emptyset$. This operation has been implemented in Mathematica for any number of vertices.

Finally, we combine (5.12) and (5.13), bearing in mind that for three given subsets, $\{i, j, k\},\{r, s\},\{t, u\}$, one has to satify,

$$
\begin{aligned}
& \{r, s\} \subseteq\{i, j, k\} \\
& \{t, u\} \cap\{i, j, k\}=\emptyset
\end{aligned} .
$$

Then, the $\mathrm{N}^{4} \mathrm{MLT}$ configuration can be expressed as follows,

$$
d \mathcal{A}_{\mathrm{N}^{4} \mathrm{MLT}}^{(L)} \sim \sum_{\substack{i=1 \\ j=i+1 \\ k=j+1}}^{6} \sum_{\substack{t=1 \\ u=t+1 \\ u, t \neq i, j, k}}^{6} L_{i j k}^{+} L_{t u}^{-},
$$

with,

$$
L_{i j k}^{ \pm}=\frac{1}{\lambda_{i j k}^{ \pm}}\left(L_{i j}^{ \pm}+L_{i k}^{ \pm}+L_{j k}^{ \pm}\right) .
$$

We remark that, in order to check the validity of this relation, we have used the collapsing procedure to recover the expression of eq. (5.14). Likewise, we use the removing procedure to generate the causal representation of the $s^{-}, t$ - and $u$-like diagrams of ref. [61].

- Similarly, for $\mathrm{N}^{5} \mathrm{MLT}, \mathrm{N}^{6} \mathrm{MLT}$, and $\mathrm{N}^{7} \mathrm{MLT}$,

$$
\begin{aligned}
& d \mathcal{A}_{\mathrm{N}^{5} \mathrm{MLT}}^{(L)} \sim \sum_{\substack{i_{1}<i_{2}<i_{3} \\
j_{1}<j_{2}<j_{3}}}^{7} \Omega_{i_{1} i_{2} i_{3}}^{j_{1} j_{2} j_{3}} L_{i_{1} i_{2} i_{3}}^{+} L_{j_{1} j_{2} j_{3}}^{-}, \\
& d \mathcal{A}_{\mathrm{N}^{6} \mathrm{MLT}}^{(L)} \sim \sum_{\substack{i_{1}<i_{2}<i_{3}<i_{4} \\
j_{1}<j_{2}<j_{3}}}^{8} \Omega_{i_{1} i_{2} i_{3} i_{4}}^{j_{1} j_{2} j_{3}} L_{i_{1} i_{2} i_{3} i_{4}}^{+} L_{j_{1} j_{2} j_{3}}^{-}, \\
& d \mathcal{A}_{\mathrm{N}^{7} \mathrm{MLT}}^{(L)} \sim \sum_{\substack{i_{1}<i_{2}<i_{3}<i_{4} \\
j_{1}<j_{2}<j_{3}<j_{4}}}^{9} \Omega_{i_{1} i_{2} i_{3} i_{4}}^{j_{1} j_{2} j_{3} j_{4}} L_{i_{1} i_{2} i_{3} i_{4}}^{+} L_{j_{1} j_{2} j_{3} j_{4}}^{-},
\end{aligned}
$$


where, in order to simplify the notation in the indices of the sum, we introduce a function, $\Omega$, that can be either 0 or 1 , depending on the intersection of the sets, $\vec{i}=\left\{i_{1}, i_{2}, \ldots, i_{N_{i}}\right\}$ and $\vec{j}=\left\{j_{1}, j_{2}, \ldots, j_{N_{j}}\right\}$,

$$
\Omega_{\vec{i}}^{\vec{j}}=\left\{\begin{array}{ll}
1 & \text { If } \vec{i} \cap \vec{j}=\emptyset \\
0 & \text { otherwise }
\end{array},\right.
$$

and,

$$
L_{i_{1} i_{2} i_{3} i_{4}}^{ \pm}=\frac{1}{\lambda_{i_{1} i_{2} i_{3} i_{4}}^{ \pm}}\left(L_{i_{1} i_{3} i_{4}}^{ \pm}+L_{i_{1} i_{2} i_{4}}^{ \pm}+L_{i_{1} i_{2} i_{3}}^{ \pm}+L_{i_{2} i_{3} i_{4}}^{ \pm}\right)
$$

Hence, with the above considerations, the extension of the causal representation of a loop topology built from $k+2$ vertices and connecting all internal lines, $\mathrm{N}^{k} \mathrm{MLT}$, can be conjectured to be,

$$
d \mathcal{A}_{\mathrm{N}^{k} \mathrm{MLT}}^{(L)} \sim \sum_{\substack{i_{1} \ll i_{N_{i}} \\ j_{1} \ll j_{N_{j}}}}^{k+2} \Omega_{\vec{i}}^{\vec{j}} L_{i_{1} i_{2} \ldots i_{N_{i}}}^{+} L_{j_{1} j_{2} \ldots j_{N_{j}}}^{-},
$$

where $i_{1} \ll i_{N_{i}}$ is the lexicographic ordering $i_{1}<i_{2}<\cdots<i_{N_{i}}$ and $N_{i}=[k / 2]+1$ and $N_{j}=k-[k / 2]$.

In eqs. (5.17), we provide the explicit causal representation for $\mathrm{N}^{4} \mathrm{MLT}, \mathrm{N}^{5} \mathrm{MLT}$, $\mathrm{N}^{6} \mathrm{MLT}$ and $\mathrm{N}^{7} \mathrm{MLT}$ without applying the LTD formalism. In fact, applying LTD on these topologies turns out to be not efficient and an unnecessary calculation, which is due to the large number of loop momenta. Let us recall that to compute the most general causal representation of these topologies, one needs to consider, as summarised in table 2 , 10, 15, 21 and 28 loop momenta, respectively. However, to cross-check our expressions, we make use of the removing procedure presented in section 3 and compare our results with the ones expected from the nested application of the residue at lower loops. To do so, we consider the following loop topologies:

- one-loop topologies with up-to nine external momenta,

- two-loop topologies with up-to seven external momenta,

- ladder triangles up-to four loops,

- three-loop tennis-court like diagram with four external momenta,

- four-loop fishnet with four external momenta.

In order to make the comparison of the general results presented in this section with the particular cases obtained by applying LTD, we focus only on the numerical value of the integrand, finding completely agreement. Additionally, to render the integrand numerically stable, we consider the energy component of the external momenta being imaginary. Thus, no singularities appear when evaluating the results obtained from LTD, which at first corresponds to individual residues containing causal and non-causal propagators, as carefully explained in ref. [60]. 


\section{Conclusions}

The novel representation of the loop-tree duality (LTD) formalism that started in ref. [59], has allowed for an understanding of an integrand representation displaying only physical information, the so-called causal representation of multi-loop Feynman integrands, introduced in ref. [60] for three master topologies: Maximal (MLT), Next-to Maximal (NMLT) and Next-to-Next-to Maximal ( $\mathrm{N}^{2} \mathrm{MLT}$ ) loop topologies. In particular, the behaviour at an arbitrary loop order was considered, yet obtaining the same representation in terms of causal propagators. In this paper, we performed a classification of these topologies by introducing the notion of the features a loop topology is characterised by: vertices and edges. While the former can be related at most as the maximum number of external legs, the latter accounts for the collection of all internal lines that connect two vertices. In effect, in this paper, we recalled the causal representation of MLT, NMLT and $\mathrm{N}^{2}$ MLT in terms of vertices and edges. We found that the results of ref. [60] can be straightforwardly be obtained from the general form of our expressions.

In view of the results of the MLT, NMLT and $\mathrm{N}^{2} \mathrm{MLT}$ configurations, with the presence of external momenta, we investigated the relations that may exit among themselves. We noted that from $\mathrm{N}^{2} \mathrm{MLT}$, being the most general topology of them, we could obtain, without performing the nested application of the LTD formalism [62], the causal representation of topologies with less edges and/or less vertices. To this end, we defined two procedures: removing and collapsing. In the removing procedure, one is interested in only removing edges by keeping fixed the number of vertices. For instance, a one-loop box was obtained by removing two edges in the causal representation of $\mathrm{N}^{2} \mathrm{MLT}$. Likewise, the collapsing procedure reduces the number of vertices, allowing, in this way, to obtain NMLT and MLT from $\mathrm{N}^{2} \mathrm{MLT}$.

Furthermore, we considered the most general topologies made of $n$ vertices, in which the number of edges accounts for all possible connections among vertices. Within the approaches presented in this paper, we extended the results of the two four-loop topologies with eight and nine propagators presented in ref. [61]. In fact, these topologies correspond to particular cases of our $\mathrm{N}^{3} \mathrm{MLT}$ and $\mathrm{N}^{4} \mathrm{MLT}$ configurations, where the removing procedure was utilised. Thus, to check our results, we used the collapsing and removing procedures to find a topology with lower complexity. In other words, by computing the most general $\mathrm{N}^{k} \mathrm{MLT}$ configuration with $k+2$ vertices and all possible edges, the generation of all $\mathrm{N}^{k-1} \mathrm{MLT}$ is straightforward.

In light of the compact and symmetric formulae of the MLT, NMLT and $\mathrm{N}^{2} \mathrm{MLT}$ configurations, we understood a pattern to generate causal representation of multi-loop topologies without making use of the LTD formalism. Following this alternative procedure, we found the causal representation of all topologies up-to $\mathrm{N}^{7} \mathrm{MLT}$, leaving, thus, a conjecture and procedure to generate any topology independently of the number of vertices.

We would be interested to see whether the approach studied in ref. [90] could efficiently generate similar results to the ones presented in this paper. Given the fact that a purely geometrical approach was applied there, a connection with the current algebraic description will be desirable. 


\section{Acknowledgments}

We are indebted to Germán Rodrigo with whom the novel application of the loop-tree duality started and for numerous insightful discussions. We also wish to thank German Sborlini for making available his results prior to publication and for comments on the manuscript. This work is supported by the COST Action CA16201 PARTICLEFACE.

Open Access. This article is distributed under the terms of the Creative Commons Attribution License (CC-BY 4.0), which permits any use, distribution and reproduction in any medium, provided the original author(s) and source are credited.

\section{References}

[1] A. Blondel, J. Gluza, S. Jadach, P. Janot and T. Riemann, eds., Theory for the FCC-ee: Report on the 11th FCC-ee Workshop Theory and Experiments, CYRM-2020-003 [arXiv: 1905.05078] [INSPIRE].

[2] P. Banerjee et al., Theory for muon-electron scattering @10 ppm: A report of the MUonE theory initiative, Eur. Phys. J. C 80 (2020) 591 [arXiv:2004.13663] [InSPIRE].

[3] C.F. Berger et al., An Automated Implementation of On-Shell Methods for One-Loop Amplitudes, Phys. Rev. D 78 (2008) 036003 [arXiv:0803.4180] [INSPIRE].

[4] F. Cascioli, P. Maierhofer and S. Pozzorini, Scattering Amplitudes with Open Loops, Phys. Rev. Lett. 108 (2012) 111601 [arXiv:1111.5206] [INSPIRE].

[5] S. Badger, B. Biedermann, P. Uwer and V. Yundin, Numerical evaluation of virtual corrections to multi-jet production in massless QCD, Comput. Phys. Commun. 184 (2013) 1981 [arXiv: 1209.0100] [INSPIRE].

[6] G. Cullen et al., GoSAm-2.0: a tool for automated one-loop calculations within the Standard Model and beyond, Eur. Phys. J. C $\mathbf{7 4}$ (2014) 3001 [arXiv:1404.7096] [INSPIRE].

[7] S. Actis, A. Denner, L. Hofer, J.-N. Lang, A. Scharf and S. Uccirati, RECOLA: REcursive Computation of One-Loop Amplitudes, Comput. Phys. Commun. 214 (2017) 140 [arXiv: 1605.01090] [INSPIRE].

[8] J. Alwall et al., The automated computation of tree-level and next-to-leading order differential cross sections, and their matching to parton shower simulations, JHEP 07 (2014) 079 [arXiv: 1405.0301] [inSPIRE].

[9] G. Heinrich, Collider Physics at the Precision Frontier, arXiv:2009.00516 [InSPIRE].

[10] D.E. Soper, QCD calculations by numerical integration, Nucl. Phys. B Proc. Suppl. 79 (1999) 444.

[11] D.E. Soper, Techniques for QCD calculations by numerical integration, Phys. Rev. D 62 (2000) 014009 [hep-ph/9910292] [INSPIRE].

[12] T. Binoth and G. Heinrich, An automatized algorithm to compute infrared divergent multiloop integrals, Nucl. Phys. B 585 (2000) 741 [hep-ph/0004013] [INSPIRE].

[13] D.E. Soper, Choosing integration points for QCD calculations by numerical integration, Phys. Rev. D 64 (2001) 034018 [hep-ph/0103262] [INSPIRE]. 
[14] M. Krämer and D.E. Soper, Next-to-leading order numerical calculations in Coulomb gauge, Phys. Rev. D 66 (2002) 054017 [hep-ph/0204113] [INSPIRE].

[15] S. Becker, C. Reuschle and S. Weinzierl, Numerical NLO QCD calculations, JHEP 12 (2010) 013 [arXiv: 1010.4187] [INSPIRE].

[16] S. Becker, C. Reuschle and S. Weinzierl, Efficiency Improvements for the Numerical Computation of NLO Corrections, JHEP 07 (2012) 090 [arXiv:1205.2096] [INSPIRE].

[17] R. Pittau, A four-dimensional approach to quantum field theories, JHEP 11 (2012) 151 [arXiv: 1208.5457] [INSPIRE].

[18] A.M. Donati and R. Pittau, Gauge invariance at work in FDR: $H \rightarrow \gamma \gamma$, JHEP 04 (2013) 167 [arXiv: 1302.5668] [INSPIRE].

[19] R.A. Fazio, P. Mastrolia, E. Mirabella and W.J. Torres Bobadilla, On the Four-Dimensional Formulation of Dimensionally Regulated Amplitudes, Eur. Phys. J. C 74 (2014) 3197 [arXiv: 1404.4783] [INSPIRE].

[20] A. Primo and W.J. Torres Bobadilla, BCJ Identities and d-Dimensional Generalized Unitarity, JHEP 04 (2016) 125 [arXiv:1602.03161] [INSPIRE].

[21] P. Mastrolia, A. Primo, U. Schubert and W.J. Torres Bobadilla, Off-shell currents and color-kinematics duality, Phys. Lett. B 753 (2016) 242 [arXiv:1507.07532] [INSPIRE].

[22] R.J. Hernandez-Pinto, G.F.R. Sborlini and G. Rodrigo, Towards gauge theories in four dimensions, JHEP 02 (2016) 044 [arXiv:1506.04617] [INSPIRE].

[23] G.F.R. Sborlini, F. Driencourt-Mangin, R. Hernandez-Pinto and G. Rodrigo, Four-dimensional unsubtraction from the loop-tree duality, JHEP 08 (2016) 160 [arXiv: 1604.06699] [INSPIRE].

[24] G.F.R. Sborlini, F. Driencourt-Mangin and G. Rodrigo, Four-dimensional unsubtraction with massive particles, JHEP 10 (2016) 162 [arXiv:1608.01584] [INSPIRE].

[25] C. Gnendiger et al., To d, or not to d: recent developments and comparisons of regularization schemes, Eur. Phys. J. C 77 (2017) 471 [arXiv:1705.01827] [INSPIRE].

[26] Z. Capatti, V. Hirschi, A. Pelloni and B. Ruijl, Local Unitarity: a representation of differential cross-sections that is locally free of infrared singularities at any order, JHEP 04 (2021) 104 [arXiv : 2010.01068] [INSPIRE].

[27] W.J. Torres Bobadilla et al., May the four be with you: Novel IR-subtraction methods to tackle NNLO calculations, Eur. Phys. J. C 81 (2021) 250 [arXiv:2012.02567] [INSPIRE].

[28] R.M. Prisco and F. Tramontano, Dual Subtractions, arXiv:2012.05012 [INSPIRE].

[29] H.A. Chawdhry, M.L. Czakon, A. Mitov and R. Poncelet, NNLO QCD corrections to three-photon production at the LHC, JHEP 02 (2020) 057 [arXiv:1911.00479] [INSPIRE].

[30] F. Caola, A. Von Manteuffel and L. Tancredi, Diphoton Amplitudes in Three-Loop Quantum Chromodynamics, Phys. Rev. Lett. 126 (2021) 112004 [arXiv:2011.13946] [INSPIRE].

[31] S. Kallweit, V. Sotnikov and M. Wiesemann, Triphoton production at hadron colliders in NNLO QCD, Phys. Lett. B 812 (2021) 136013 [arXiv:2010.04681] [INSPIRE].

[32] S. Badger, H.B. Hartanto and S. Zoia, Two-loop QCD corrections to Wbb production at hadron colliders, arXiv:2102.02516 [INSPIRE]. 
[33] B. Agarwal, F. Buccioni, A. von Manteuffel and L. Tancredi, Two-loop leading colour QCD corrections to $q \bar{q} \rightarrow \gamma \gamma g$ and $q g \rightarrow \gamma \gamma q$, arXiv:2102.01820 [INSPIRE].

[34] K.G. Chetyrkin and F.V. Tkachov, Integration by Parts: The Algorithm to Calculate $\beta$-functions in 4 Loops, Nucl. Phys. B 192 (1981) 159 [INSPIRE].

[35] A.V. Kotikov, Differential equation method: The Calculation of $N$ point Feynman diagrams, Phys. Lett. B 267 (1991) 123 [Erratum ibid. 295 (1992) 409] [InSPIRE].

[36] S. Laporta, High precision calculation of multiloop Feynman integrals by difference equations, Int. J. Mod. Phys. A 15 (2000) 5087 [hep-ph/0102033] [inSPIRE].

[37] A. von Manteuffel and C. Studerus, Reduze 2 - Distributed Feynman Integral Reduction, arXiv: 1201.4330 [INSPIRE].

[38] J.M. Henn, Multiloop integrals in dimensional regularization made simple, Phys. Rev. Lett. 110 (2013) 251601 [arXiv:1304.1806] [INSPIRE].

[39] M. Argeri et al., Magnus and Dyson Series for Master Integrals, JHEP 03 (2014) 082 [arXiv:1401.2979] [INSPIRE].

[40] P. Maierhöfer, J. Usovitsch and P. Uwer, Kira - A Feynman integral reduction program, Comput. Phys. Commun. 230 (2018) 99 [arXiv:1705.05610] [InSPIRE].

[41] S. Borowka, G. Heinrich, S.P. Jones, M. Kerner, J. Schlenk and T. Zirke, SecDec-3.0: numerical evaluation of multi-scale integrals beyond one loop, Comput. Phys. Commun. 196 (2015) 470 [arXiv:1502.06595] [INSPIRE].

[42] A.V. Smirnov, FIESTA4: Optimized Feynman integral calculations with GPU support, Comput. Phys. Commun. 204 (2016) 189 [arXiv:1511.03614] [INSPIRE].

[43] K.J. Larsen and Y. Zhang, Integration-by-parts reductions from unitarity cuts and algebraic geometry, Phys. Rev. D 93 (2016) 041701 [arXiv:1511.01071] [INSPIRE].

[44] P. Mastrolia and S. Mizera, Feynman Integrals and Intersection Theory, JHEP 02 (2019) 139 [arXiv: 1810.03818] [INSPIRE].

[45] S. Abreu et al., Caravel: A C++ Framework for the Computation of Multi-Loop Amplitudes with Numerical Unitarity, arXiv:2009.11957 [INSPIRE].

[46] H. Ita, Two-loop Integrand Decomposition into Master Integrals and Surface Terms, Phys. Rev. D 94 (2016) 116015 [arXiv: 1510.05626] [INSPIRE].

[47] S. Catani, T. Gleisberg, F. Krauss, G. Rodrigo and J.-C. Winter, From loops to trees by-passing Feynman's theorem, JHEP 09 (2008) 065 [arXiv: 0804.3170] [INSPIRE].

[48] I. Bierenbaum, S. Catani, P. Draggiotis and G. Rodrigo, A Tree-Loop Duality Relation at Two Loops and Beyond, JHEP 10 (2010) 073 [arXiv: 1007.0194] [INSPIRE].

[49] I. Bierenbaum, S. Buchta, P. Draggiotis, I. Malamos and G. Rodrigo, Tree-Loop Duality Relation beyond simple poles, JHEP 03 (2013) 025 [arXiv:1211.5048] [INSPIRE].

[50] S. Buchta, G. Chachamis, P. Draggiotis, I. Malamos and G. Rodrigo, On the singular behaviour of scattering amplitudes in quantum field theory, JHEP 11 (2014) 014 [arXiv: 1405.7850] [INSPIRE].

[51] S. Buchta, G. Chachamis, P. Draggiotis and G. Rodrigo, Numerical implementation of the loop-tree duality method, Eur. Phys. J. C 77 (2017) 274 [arXiv:1510.00187] [INSPIRE]. 
[52] C. Bogner and S. Weinzierl, Feynman graph polynomials, Int. J. Mod. Phys. A 25 (2010) 2585 [arXiv: 1002.3458] [INSPIRE].

[53] J.L. Jurado, G. Rodrigo and W.J. Torres Bobadilla, From Jacobi off-shell currents to integral relations, JHEP 12 (2017) 122 [arXiv:1710.11010] [INSPIRE].

[54] F. Driencourt-Mangin, G. Rodrigo and G.F.R. Sborlini, Universal dual amplitudes and asymptotic expansions for $g g \rightarrow H$ and $H \rightarrow \gamma \gamma$ in four dimensions, Eur. Phys. J. $C \mathbf{7 8}$ (2018) 231 [arXiv: 1702.07581] [INSPIRE].

[55] F. Driencourt-Mangin, G. Rodrigo, G.F.R. Sborlini and W.J. Torres Bobadilla, Universal four-dimensional representation of $H \rightarrow \gamma \gamma$ at two loops through the Loop-Tree Duality, JHEP 02 (2019) 143 [arXiv: 1901.09853] [INSPIRE].

[56] F. Driencourt-Mangin, G. Rodrigo, G.F.R. Sborlini and W.J. Torres Bobadilla, On the interplay between the loop-tree duality and helicity amplitudes, arXiv:1911.11125 [INSPIRE].

[57] J. Plenter and G. Rodrigo, Asymptotic expansions through the loop-tree duality, arXiv: 2005.02119 [INSPIRE].

[58] J.J. Aguilera-Verdugo et al., Causality, unitarity thresholds, anomalous thresholds and infrared singularities from the loop-tree duality at higher orders, JHEP 12 (2019) 163 [arXiv: 1904.08389] [INSPIRE].

[59] J.J. Aguilera-Verdugo et al., Open Loop Amplitudes and Causality to All Orders and Powers from the Loop-Tree Duality, Phys. Rev. Lett. 124 (2020) 211602 [arXiv:2001.03564] [INSPIRE].

[60] J.J. Aguilera-Verdugo, R.J. Hernandez-Pinto, G. Rodrigo, G.F.R. Sborlini and W.J. Torres Bobadilla, Causal representation of multi-loop Feynman integrands within the loop-tree duality, JHEP 01 (2021) 069 [arXiv:2006.11217] [INSPIRE].

[61] S. Ramírez-Uribe, R.J. Hernández-Pinto, G. Rodrigo, G.F.R. Sborlini and W.J. Torres Bobadilla, Universal opening of four-loop scattering amplitudes to trees, JHEP 04 (2021) 129 [arXiv:2006.13818] [INSPIRE].

[62] J. Jesús Aguilera-Verdugo, R.J. Hernández-Pinto, G. Rodrigo, G.F.R. Sborlini and W.J. Torres Bobadilla, Mathematical properties of nested residues and their application to multi-loop scattering amplitudes, JHEP 02 (2021) 112 [arXiv:2010.12971] [INSPIRE].

[63] E.T. Tomboulis, Causality and Unitarity via the Tree-Loop Duality Relation, JHEP 05 (2017) 148 [arXiv:1701.07052] [INSPIRE].

[64] R. Runkel, Z. Ször, J.P. Vesga and S. Weinzierl, Integrands of loop amplitudes within loop-tree duality, Phys. Rev. D 101 (2020) 116014 [arXiv:1906.02218] [InSPIRE].

[65] R. Runkel, Z. Szőr, J.P. Vesga and S. Weinzierl, Causality and loop-tree duality at higher loops, Phys. Rev. Lett. 122 (2019) 111603 [Erratum ibid. 123 (2019) 059902] [arXiv: 1902.02135] [INSPIRE].

[66] Z. Capatti, V. Hirschi, D. Kermanschah, A. Pelloni and B. Ruijl, Manifestly Causal Loop-Tree Duality, arXiv:2009.05509 [INSPIRE].

[67] Z. Capatti, V. Hirschi, D. Kermanschah, A. Pelloni and B. Ruijl, Numerical Loop-Tree Duality: contour deformation and subtraction, JHEP 04 (2020) 096 [arXiv:1912.09291] [INSPIRE]. 
[68] Z. Capatti, V. Hirschi, D. Kermanschah and B. Ruijl, Loop-Tree Duality for Multiloop Numerical Integration, Phys. Rev. Lett. 123 (2019) 151602 [arXiv:1906.06138] [INSPIRE].

[69] L. de la Cruz, A scattering amplitudes approach to hard thermal loops, arXiv:2012.07714 [INSPIRE].

[70] O. Steinmann, Über den Zusammenhang Zwischen den Wightmanfunktionen und den Retardierten Kommutatoren, Helv. Phys. Acta 33 (1960) 257.

[71] O. Steinmann, Wightman-Funktionen und Retardierte Kommutatoren. II, Helv. Phys. Acta 33 (1960) 347.

[72] H. Araki, Generalized Retarded Functions and Analytic Function in Momentum Space in Quantum Field Theory, J. Math. Phys. 2 (1961) 163.

[73] D. Ruelle, Connection between Wightman Functions and Green Functions in p-Space, Nuovo Cim. 19 (1961) 356.

[74] H.P. Stapp, Inclusive cross-sections are discontinuities, Phys. Rev. D 3 (1971) 3177 [INSPIRE].

[75] M. Lassalle, Analyticity Properties Implied by the Many-Particle Structure of the N Point Function in General Quantum Field Theory. 1. Convolution of $n$ Point Functions Associated with a Graph, Commun. Math. Phys. 36 (1974) 185 [InSPIRE].

[76] K.E. Cahill and H.P. Stapp, Optical theorems and steinmann relations, Annals Phys. 90 (1975) 438 [INSPIRE].

[77] S. Caron-Huot, L.J. Dixon, A. McLeod and M. von Hippel, Bootstrapping a Five-Loop Amplitude Using Steinmann Relations, Phys. Rev. Lett. 117 (2016) 241601 [arXiv: 1609.00669] [INSPIRE].

[78] S. Caron-Huot, L.J. Dixon, F. Dulat, M. Von Hippel, A.J. McLeod and G. Papathanasiou, The Cosmic Galois Group and Extended Steinmann Relations for Planar $\mathcal{N}=4 S Y M$ Amplitudes, JHEP 09 (2019) 061 [arXiv:1906.07116] [INSPIRE].

[79] P. Benincasa, A.J. McLeod and C. Vergu, Steinmann Relations and the Wavefunction of the Universe, Phys. Rev. D 102 (2020) 125004 [arXiv:2009.03047] [INSPIRE].

[80] S. Caron-Huot et al., The Steinmann Cluster Bootstrap for $N=4$ Super Yang-Mills Amplitudes, PoS CORFU2019 (2020) 003 [arXiv:2005.06735] [InSPIRE].

[81] J.L. Bourjaily, H. Hannesdottir, A.J. McLeod, M.D. Schwartz and C. Vergu, Sequential Discontinuities of Feynman Integrals and the Monodromy Group, JHEP 01 (2021) 205 [arXiv: 2007.13747] [INSPIRE].

[82] A. von Manteuffel and R.M. Schabinger, A novel approach to integration by parts reduction, Phys. Lett. B $\mathbf{7 4 4}$ (2015) 101 [arXiv:1406.4513] [INSPIRE].

[83] T. Peraro, Scattering amplitudes over finite fields and multivariate functional reconstruction, JHEP 12 (2016) 030 [arXiv:1608.01902] [INSPIRE].

[84] T. Peraro, FiniteFlow: multivariate functional reconstruction using finite fields and dataflow graphs, JHEP 07 (2019) 031 [arXiv: 1905.08019] [INSPIRE].

[85] J. Klappert and F. Lange, Reconstructing rational functions with FireFly, Comput. Phys. Commun. 247 (2020) 106951 [arXiv: 1904.00009] [INSPIRE].

[86] M. Heller and A. von Manteuffel, MultivariateApart: Generalized Partial Fractions, arXiv: 2101.08283 [INSPIRE]. 
[87] W. Decker, G.-M. Greuel, G. Pfister and H. Schönemann, Singular 4-2-0 - A computer algebra system for polynomial computations, http://www. singular.uni-kl.de (2020).

[88] J. Boehm, M. Wittmann, Z. Wu, Y. Xu and Y. Zhang, IBP reduction coefficients made simple, JHEP 12 (2020) 054 [arXiv:2008.13194] [INSPIRE].

[89] W.J. Torres Bobadilla, Lotty - The loop-tree duality automation, arXiv:2103.09237 [INSPIRE].

[90] G.F.R. Sborlini, A geometrical approach to causality in multi-loop amplitudes, arXiv:2102 . 05062 [INSPIRE]. 